\section{Commentary: Publish or perish at our collective peril}

\author{
Jennie H. Kwon, MD, and Chadrick E. Denlinger, MD
}

Publishing investigative findings is a time-honored means of sharing knowledge and facilitating collective scientific advancement. For these reasons, scientific publications remain the backbone of scientific communication. Perversely, publications also function as a crucial metric of success in the academic community because of their direct links to notoriety, academic advancement, and recognition by extramural funding agencies and are responsible for the publish-or-perish mindset pervasive in the academic community that incentivizes unscrupulous publication.

D'Souza and colleagues ${ }^{1}$ examine ways to ensure research integrity in an environment facing increasing transgressions. ${ }^{1}$ The authors note examples of large fines incurred by institutions for data fabrication and other egregious forms of research misconduct. More common but less harmful manifestations of misconduct include duplication of publications and lengthy lists of authors lacking any meaningful connection to the published work. The time necessary to detect and correct false information is long enough that reported findings may already be disseminated and adopted into clinical practice before any action is taken. The authors suggest avenues to promote research integrity that focus on checks at multiple levels, including individual investigators, institutions, funding agencies, and journals.

In addition to fortifying departmental and institutional research policies, we suggest considering the incentive structures that underlie a culture that emphasizes publishing at the expense of scientific integrity. Dismantling the publish-or-perish mindset would require an institutional restructuring of career incentives away from publication quantity with a greater focus on quality. Establishing institutional value of an individual's research, regardless of

\footnotetext{
From the Division of Cardiothoracic Surgery, Department of Surgery, Medical University of South Carolina, Charleston, SC.

Disclosure: Authors have nothing to disclose with regard to commercial support.

Received for publication Feb 2, 2020; accepted for publication Feb 4, 2020; available ahead of print Feb 14, 2020.

Address for reprints: Chadrick E. Denlinger, MD, Division of Cardiothoracic Surgery,

Department of Surgery, Medical University of South Carolina, 114 Doughty St,

Charleston, SC 29425 (E-mail: denlinge@musc.edu).

J Thorac Cardiovasc Surg 2020;160:735-6

$0022-5223 / \$ 36.00$

Copyright (c) 2020 by The American Association for Thoracic Surgery

https://doi.org/10.1016/j.jtcvs.2020.02.024
}

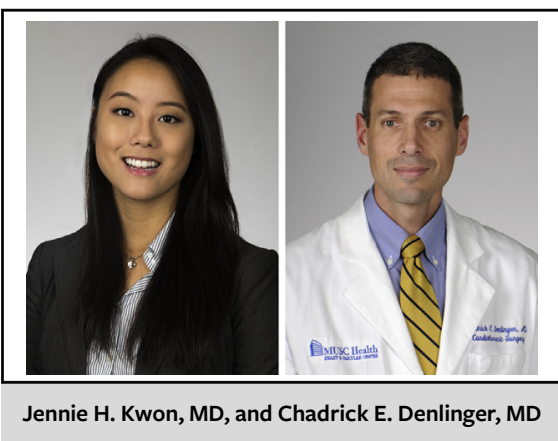

\section{CENTRAL MESSAGE \\ The publish-or-perish mindset in the academic community incen- tivizes publication of meaningless articles, and worse, fraudulent ones.}

whether or not it is published, may restructure incentives emphasizing quality rather than volume.

Journals, currently biased toward publishing positive findings rather than negative results, also bear some responsibility by encouraging investigators to produce results supporting their hypothesis for the work to make it to print. Similarly, submitted manuscripts with novel concepts rather than those verifying and confirming results published by others are more likely to be accepted for publication. These biases are likely driven by an editor's quest for a stronger impact factor, realizing that positive results are more likely to be cited than negative ones. Collectively, these factors encourage authors to submit novel and positive findings to be considered for publication.

A final avenue that might disrupt the publish-or-perish mindset could be to facilitate additional research funding through nongovernmental agencies. Without a publication track record, an investigator is unlikely to receive government funding. Therefore, interactions among smaller funding agencies, industry, and investigators may facilitate a more nimble system where agencies could provide smaller, perhaps incremental, grants for ongoing research while bypassing the requisite publications to be competitive for government agency funding. Even in this more nimble model, incentives for producing positive results are thinly veiled, highlighting the need for research verification by completely independent research groups and funding sources.

Persistence of the publish-or-perish mindset, a dominant contributor for lack of research integrity and publication of 
meaningless articles, implies there are no simple solutions. The article by D'Souza and colleagues ${ }^{1}$ is a starting point for discussions of how to minimize its influence.

\section{Reference}

1. D'Souza DM, Sade RM, Moffatt-Bruce SD. The many facets of research integrity: what can we do to ensure it? J Thorac Cardiovasc Surg. 2020;160:730-3. 